\title{
Der Einbruch der Geschichte in das geschlossene Universum der Ethnologie
}

\author{
Ein Kommentar zu Carola Lentz: „Der Kampf um die Kultur: Zur Ent- und \\ Re-Soziologisierung eines ethnologischen Konzepts“"
}

\section{Von Klaus Lichtblau}

Die im Laufe des 20. Jahrhunderts erfolgte Entstehung und Entwicklung eines genuin soziologischen Kulturverständnisses verdankt sich vieler Motive und kann auch nicht als eine geradlinig verlaufende Erfolgsgeschichte erzählt werden. Zahlreiche Brechungen verdanken sich dabei nicht nur den unterschiedlichen disziplinären Einflüssen, denen sich die Soziologie bis heute ausgesetzt sieht, sondern auch den unterschiedlichen nationalen Traditionen, die ihre Entwicklung im Laufe des 20. Jahrhunderts geprägt haben. Auch eine ,kosmopolitische Soziologie', die sich eher an der Emergenz einer, Weltgesellschaft' als an der Existenz der einzelnen Nationalstaaten orientiert, muss Auskunft darüber geben, ob sie den Kulturbegriff eher im Singular oder im Plural zu gebrauchen beabsichtigt und welchen Status sie dabei der ,Kultur' bzw. den ,Kulturen' im Verhältnis zur ,Gesellschaft' bzw. den ,Gesellschaften' einzuräumen gedenkt.

Eine der Disziplinen, die bei der Entwicklung der verschiedenen Varianten eines soziologischen Kulturbegriffs eine nicht unerhebliche Rolle gespielt hat, ist die Ethnologie bzw. die Kulturanthropologie. Ihre Fachgeschichte überschneidet sich in vielerlei Hinsicht mit der der modernen Soziologie, auch wenn namhafte Vertreter beider Disziplinen immer wieder deren Eigenart und Autonomie hervorgehoben haben. Immerhin sind die grundbegrifflichen Probleme beider Disziplinen sehr ähnlich, wenn es um das Verhältnis der ,Gesellschaft' zur ,Kultur' bzw. ,Zivilisation' geht. Die im englischen und französischen Sprachraum vorherrschende Gleichsetzung von ,Zivilisation' und ,Kultur' hat nämlich immer wieder dazu Anlass gegeben, einen Kulturbegriff kritisch zu hinterfragen, der letztlich mit dem der, Gesellschaft' identisch ist. Denn als der britische Anthropologe und Religionswissenschaftler Edward Burnett Tylor 1871 in seinem einflussreichen Buch Primitive Culture den Bereich der Kultur als ,that complex whole“ definierte, ,which includes knowledge, belief, art, law, morals, customs, and any other capabilities and habits acquired by man as a member of society“ (1871: 1), brachte er damit ein extrem weit gefasstes Kulturverständnis zum Ausdruck, unter das im Grunde genommen alles subsumiert werden konnte, was andere Autoren als Eigenart des gesellschaftlichen Lebens der Menschen ansahen. Die in der Ethnologie und Kulturanthropologie vorherrschende Gleichsetzung von ,Kultur' und ,Gesellschaft' wird auch nicht dadurch relativiert, dass das Wort ,Kultur' prinzipiell im Plural gebraucht wird, sondern im Gegenteil. Denn auch in diesem Fall wird die Eigenart einer Gemeinschaft bzw. einer Gesellschaft auf deren ,Kultur' zurückgeführt, was unweigerlich zu einer Reifizierung und Essentialisierung des ethnologischen Kulturbegriffs führen muss. Insofern ist der an bestimmte Richtungen der Ethnologie adressierte Vorwurf des ,Kulturrelativismus' missverständlich. Denn auch die Vorstellung, dass wir es prinzipiell mit verschiedenen ,Kulturkreisen' zu tun haben, die jeweils das menschliche Leben prägen, unterstellt ja gerade die Möglichkeit der Identifizierung einer ethnischen Gruppe bzw. ,Nation' durch deren kulturelle Eigenart. Eine Pluralisierung des Kulturbegriffs führt in diesem Fall also nicht weiter, ihre Vermeidung aber ebenfalls nicht, um seine reflektierte ,Soziologisierung' bzw. eine entsprechende ,Kulturalisierung' des Gesellschaftsbegriffs zu bewerkstelligen.

Genau vor diesem Dilemma standen Alfred Kroeber und Talcott Parsons, als sie 1958 in einem viel beachteten Statement den Vorschlag machten, eine sowohl für Soziologen als auch 
für Ethnologen verbindliche analytische Unterscheidung zwischen dem Begriff der, Gesellschaft' und dem der ,Kultur' vorzunehmen. Sie empfahlen in diesem Zusammenhang, den ersteren Begriff auf den Bereich der sozialen Interaktionen und ihre Strukturen (patterns of interaction), letzteren dagegen auf die Sphäre der symbolischen Bedeutungen zu beschränken, wobei in diesem Fall das Spektrum von der Kunst über die Literatur und die Philosophie bis zur Religion reicht (vgl. Kroeber / Parsons 1958). ${ }^{1}$ Parsons und Kroeber gingen dabei von der Feststellung aus, dass an den sozialwissenschaftlichen Departments der USA die Anthropologen zu dieser Zeit den Kulturbegriff in derselben universalistischen Weise zur Kennzeichnung eines Sachverhaltes gebrauchten, den die Soziologen als , Gesellschaft' bezeichneten. Ihr Vorschlag, eine entsprechende Arbeitsteilung zwischen Ethnologie und Soziologie dergestalt vorzunehmen, dass erstere für den Bereich der Kultur, letztere dagegen für die Gesellschaft bzw. das ,Social System' zuständig sei, kann allerdings insofern nicht ganz ernst gemeint gewesen sein, als Parsons natürlich nicht vorhatte, einer ,Entsoziologisierung' des Kulturbegriffs einzuleiten, wie dies Carola Lentz in ihrem Essay „Der Kampf um die Kultur“ anzunehmen scheint. Vielmehr spielte die ,Kultur' bzw. das entsprechende gesellschaftliche, Treuhandsystem' Parsons zufolge eine zentrale Rolle innerhalb der Entstehung und der Entwicklung des ,Systems moderner Gesellschaften', wie jeder Kenner seines auch heute noch überaus lesenswerten Buches gleichnamigen Titels weiß. ${ }^{2}$

Die von Carola Lentz in sehr sympathischer und instruktiver Weise wieder angestoßene Diskussion über den Gebrauch des Begriffs ,Kultur' in der Ethnologie und der Soziologie sollte deshalb primär als Beitrag zum Versuch einer Rettung des Kulturbegriffs innerhalb der Ethnologie verstanden werden, der in den letzten Jahrzehnten aus vielen Gründen in die Dauerkritik geraten ist, nicht aber als Beitrag zur Geschichte des soziologischen Kulturbegriffs, auch wenn sich letztere immer wieder mit der Geschichte des ethnologischen Kulturbegriffs gekreuzt bzw. überlappt hat. Was kann man aber als Soziologe von der von ihr erzählten Geschichte über die Ent- und Resoziologisierung des ethnologischen Kulturverständnisses eigentlich lernen? Nun: Immerhin soviel, dass offensichtlich nicht nur die Soziologie, sondern auch die Ethnologie nach wie vor große Probleme damit hat, das Verhältnis zwischen, Gesellschaft' und ,Kultur' so auszubuchstabieren, dass die mit dieser analytischen Unterscheidung verbundenen Aporien endlich zufriedenstellend aufgelöst werden können.

Carola Lentz spricht sich in diesem Zusammenhang im Anschluss an Andreas Reckwitz für eine ,praxistheoretische' Reformulierung des Kulturbegriffs aus, um diesen als ein interdisziplinäres ,Brückenkonzept' zu rehabilitieren. Ich habe in diesem Zusammenhang allerdings nicht ganz verstanden, ob ihrer Meinung nach nicht nur in der Ethnologie, sondern auch in der Soziologie ein Bedarf hierfür besteht. Zumindest halte ich ihre Annahme für fraglich, dass mit den definitorischen Vorschlägen von Parsons und Kroeber der Weg für eine „,gesellschaftsfreie Definition von Kultur, die Konzentration auf Werte und Weltbilder statt Handlungen und Institutionen sowie die Abstraktion von Materialität und Macht" frei gemacht worden sei. Diesen

$1 \mathrm{Zu}$ der sich unmittelbar daran anschließenden Kontroverse, die insbesondere den wissenschaftslogischen Gehalt der von Parsons und Kroeber als , analytisch' verstandenen Unterscheidung zwischen Kultur und Gesellschaft betraf, vgl. die einzelnen Stellungnahmen in der American Sociological Review 24 (1959), S. 246-250.

2 Vgl. Talcott Parsons, Das System moderner Gesellschaften, München 1972. Diesem Buch ging ein weiteres, im Englischen unter dem Titel Societies erschienenes Buch voraus, in dem er im Anschluß an Max Weber die Bedeutung von epochalen kulturellen Neuerungen wie die Entstehung der antiken griechischen Philosophie und der hebräischen Prophetie für die gesamtgesellschaftliche Entwicklung des ,Okzidents' ausdrücklich hervorhob. Auch wenn Parsons seinem eigenen Selbstverständnis nach ein ,kultureller Determinist' war, heißt dies noch lange nicht, daß er einer Entsoziologisierung des Kulturbegriffs Vorschub leistete und diesen dabei der Ethnologie überließ. Oder fühlt sich letztere inzwischen auch für die Entwicklung der, abendländischen Kultur' in den letzten 2600 Jahren zuständig? 
Vorwurf hätte sie nämlich genau so gut gegenüber Parsons' Gewährsautor Max Weber richten können, der bekanntlich das Wechselspiel zwischen den ,Ideen' und den ,Interessen' in den Mittelpunkt seiner kultur- und universalgeschichtlichen Studien stellte, dabei jedoch gerade nicht von der Einbettung der ,Kulturinhalte' und ,Weltbilder' in übergreifende ökonomische, politische und soziale Zusammenhänge abstrahierte. Allerdings sprach er ihnen in bestimmten Fällen auch die Funktion einer, Weichenstellung' zu, die sich ihm zufolge aus bestimmten Eigengesetzlichkeiten innerhalb der Entwicklung des Rationalismus und Intellektualismus ergebe (vgl. Weber 1920: 252). Keineswegs war damit jedoch eine idealistische Geschichtsphilosophie im hegelschen Sinne anvisiert und auch keine Reduktion der kulturellen auf die gesellschaftliche Sphäre intendiert, sondern die Betonung ihrer wechselseitigen relativen Autonomie. Eine ,machttheoretische' Interpretation des Kulturbegriffs war Max Weber, der bekanntlich dem ,Kampf' den Status eines soziologischen Grundbegriffs zusprach, insofern mit Sicherheit nicht fremd; allerdings betonte er in diesem Zusammenhang, dass es neben dem "offenen und instabilen Prozeß des Aushandelns von Bedeutungen" (Lentz) in der kulturellen Wertsphäre auch noch so etwas wie ein ,Gebot der Konsequenz' gebe, das die relative Autonomie der Ideenentwicklung gegenüber entsprechenden politischen, ökonomischen und sozialen Funktionalisierungsversuchen unterstreiche (vgl. Lichtblau 2009: 69ff).

Kurz und gut: Ich halte die von Carola Lentz und anderen eingeklagte macht- und handlungstheoretische Reformulierung des Kulturbegriffs für eine soziologische Selbstverständlichkeit, die auch in dem Prestige zum Ausdruck kommt, welches das Werk von Pierre Bourdieu heute zu Recht weltweit genießt. Ich hätte auch den italienischen Marxisten Antonio Gramsci zitieren können, der in seinen Kerkerheften die geschichtliche Bedeutung des Kampfes des Bürgertums und der Arbeiterbewegung um die kulturelle Hegemonie so eindrucksvoll beschrieben hat (vgl. 1967: 282ff.; 1972). Und selbst das von Friedrich Tenbruck vertretene Verständnis von , bürgerlicher Kultur' betont ja gerade die Rolle der Lesegesellschaften, Clubs und Vereine sowie der verschiedenen Arten der bürgerlichen Intelligenz für die Entstehung und Entwicklung dieser sehr spezifischen Art der ,kulturellen Vergesellschaftung' (vgl. 1989: $251 \mathrm{ff})$.

Die Annahme einer relativen Verselbständigung der Kultur und der Einbettung dieses Prozesses in umfassendere soziale Konflikte, Kämpfe und Strukturen einer Epoche stellt also zumindest innerhalb der Soziologie einen Gemeinplatz dar. Daß diese Einsicht inzwischen auch innerhalb der Ethnologie eine weite Verbreitung gefunden hat, kann der hierfür sehr einschlägigen Darstellung von Carola Lentz entnommen werden, die mir diesbezüglich vorbildlich zu sein scheint. Die von ihr in diesem Zusammenhang immer wieder beschworene Gefahr, innerhalb der Ethnologie die „Symbolsysteme als autonome Gebilde von historisch spezifischen, politischen Prozessen der Machtbildung abzukoppeln“, ist jedoch mit Sicherheit nicht die logische Konsequenz der von Parsons und Kroeber empfohlenen Arbeitsteilung zwischen Soziologie und Ethnologie, sondern Ausdruck der scheinbaren Zeitlosigkeit archaischer Gesellschaften, wie sie innerhalb einer bestimmten Richtung der ethnologischen Literatur und der ihr zugrunde liegenden Rekonstruktionslogik zum Ausdruck kommt. Der Einbruch der Geschichte in das vermeintlich geschlossene Universum vormoderner Kulturen war insofern in jeder Hinsicht lehrreich, auch wenn sich dieser gerade für letztere meist desaströs ausgewirkt hat. 


\section{Literatur}

Gramsci, Antonio (1967): Philosophie der Praxis. Eine Auswahl, Frankfurt / Main.

Gramsci, Antonio (1972): Briefe aus dem Kerker, Frankfurt / Main.

Kroeber, Alfred L. / Talcott Parsons (1958): The Concepts of Culture and of Social System, in: American Sociological Review 23, S. 582-583.

Lichtblau, Klaus (2009): Sprachwandel und Gesellschaftswandel. Zur historischen Semantik von epochenbegriffen, in: Gerhard Preyer (Hrsg.), Neuer Mensch und kollektive Identität in der Kommunikationsgesellschaft, Wiesbaden, S. 69-87.

Tenbruck, Friedrich (1989): Die kulturellen Grundlagen der Gesellschaft, Opladen.

Tylor, Edward B. (1871): Primitive Culture. Researches into the Development of Mythology, Philosophy, Religion, Art, and Custom, London.

Weber, Max (1920): Gesammelte Aufsätze zur Religionssoziologie, Band 1, Tübingen.

Prof. Dr. Klaus Lichtblau Johann Wolfgang Goethe Universität Frankfurt am Main Institut für Gesellschafts- und Politikanalyse

Robert-Mayer-Str. 5 60054 Frankfurt am Main k.lichtblau@soz.uni-frankfurt.de 